\title{
Calidad docente, formación y evaluación de estudiantes universitarios: editorial
}

\section{Teaching Quality, Training and Assessment of University Students: Editorial}

\author{
Mauricio González-Arias ${ }^{\mathrm{a}}$ \& Agustín Martínez-Molina ${ }^{\mathrm{b}}$ \\ ${ }^{a}$ Universidad de La Serena, La Serena, Chile \\ ${ }^{b}$ Universidad de Talca, Talca, Chile
}

Chile está bien posicionado en los rankings internacionales de universidades. En 2016 una veintena de universidades chilenas clasificó en estas listas (e.g., QS World University Rankings, Times Higher Education World University Rankings). Dos instituciones (Universidad de Chile y Pontificia Universidad Católica de Chile) se situaron entre las 10 primeras a nivel latinoamericano, y por encima de la mitad de la tabla a nivel mundial. En investigación específicamente, Chile ocupa la $4^{\mathrm{a}}$ posición en Latinoamérica (después de Brasil, México y Argentina), y la número 38 de 239 países a nivel mundial (Centil 84 según el índice h total consultado en SCImago, 2016). Sin entrar a discutir las discrepancias y falta de propiedades psicométricas de los indicadores que alimentan estas listas o sistemas (Aguillo, Bar-Ilan, Levene, \& Ortega, 2010; Bookstein, Seidler, Fieder, \& Winckler, 2010), se puede argumentar con estos números que Chile tiene, en general, un sistema de educación superior de calidad aunque mejorable.

En Latinoamérica, poco a poco, la calidad de los procesos de formación (e.g., competencias) en carreras de pregrado se ha vuelto materia relevante (Asún, Zúñiga, \& Ayala, 2013; Juliá, 2013). El rápido cambio en el acceso, la masificación, la necesidad de acortar el tiempo total de la formación profesional, la necesidad de identificar e intervenir en las causas de retraso en la titulación y en la deserción, por una parte, y la desregulación de la oferta en la formación de nuevos profesionales sin adecuados controles de calidad de los procesos de formación, por otra, generan importantes desafíos a las instituciones de formación superior y en particular a la psicología (Urzúa, Vera-Villarroel, Zúñiga, \& Salas, 2015).

Para enfrentar estos desafíos no basta solo con reflexionar profundamente sobre los métodos docentes tradicionales (i.e., magistrales) en el contexto de la formación profesional y su pertinencia respecto a los cambios de la sociedad actual (e.g., amplificado con nuevas tecnologías y técnicas de evaluación). Tampoco es suficiente la consideración rigurosa de los aspectos éticos asociados a cada uno de los niveles y actores del proceso.

Para enfrentar estos desafíos se requiere fundamentalmente de investigación científica entendida en un sentido amplio. Una actividad científica que permita establecer la efectividad de nuevas y antiguas prácticas docentes, que permita validar itinerarios y objetivos de formación más allá del sentido común, y que permita estimar la fiabilidad y validez de las medidas en los distintos niveles del proceso (González-Arias, Carabantes, \& Muñoz-Carreño, 2016).

Cómo citar: González-Arias, M. \& Martínez-Molina, A. (2016). Calidad docente, formación y evaluación de estudiantes universitarios: editorial. Revista de Psicología, 25(2), 1-3. 
Las evaluaciones de calidad docente tienen ya demasiadas e importantes funciones en las universidades como para seguir sin reportar públicamente sus garantías científicas de validez o fiabilidad (Martínez-Molina \& Nistor, 2016).

Esta sección presenta cuatro trabajos que contribuyen a resolver parte de la problemática antes descrita. El primero desarrolla un modelo que consigue explicar la atención de los estudiantes en el aula (Sánchez-Rosas \& Esquivel, 2016). El modelo considera algunas de las variables que de forma sustantiva terminan incidiendo en el rendimiento académico. El segundo trabajo explora un método docente metacognitivo usado como estrategia de enseñanza reflexiva para el desarrollo de competencias del estudiante (Olate \& Castillo, 2016). El tercer trabajo profundiza en una de las últimas variables que sugiere la literatura científica, la autoeficacia, que además de las clásicas cognitivas (e.g., inteligencia, responsabilidad), contribuye a explicar el rendimiento académico universitario (Rivera Heredia, Martínez Fuentes, González Betanzos, \& Salazar García, 2016). Y el último trabajo seleccionado para esta ocasión se centra en la construcción de pruebas de conocimiento teórico para estudiantes universitarios desde la teoría de respuesta al ítem (Cupani et al., 2016).

Agradecemos a la Revista de Psicología la iniciativa y su apoyo en esta sección temática. Esperamos que estos trabajos sean de agrado y buen criterio para sus lectores.

\section{Referencias}

Aguillo, I. F., Bar-Ilan, J., Levene, M., \& Ortega, J. L. (2010). Comparing university rankings. Scientometrics, 85(1), 243-256.

http://dx.doi.org/10.1007/s11192-010-0190-z

Asún, R., Zúñiga, C., \& Ayala, M. C. (2013). La formación por competencias y los estudiantes: confluencias y divergencias en la construcción del docente ideal. Calidad en la Educación, 38, 277-304.

http://dx.doi.org/10.4067/S0718-45652013000100008

Bookstein, F. L., Seidler, H., Fieder, M., \& Winckler, G. (2010). Too much noise in the Times Higher Education rankings. Scientometrics, 85(1), 295-299. http://dx.doi.org/10.1007/s11192-010-0189-5

Cupani, M., Ghio, F. B., Leal, M. F., Giraudo, G. M., Castro Zamparella, T., Piumatti, G.,... Barrionuevo, L. (2016). Desarrollo de un banco de ítems para medir conocimiento en estudiantes universitarios. Revista de Psicología, 25(2), 1-19.

http://dx.doi.org/10.5354/0719-0581.2017.44808

González-Arias, M., Carabantes, E., \& Muñoz-Carreño, N. (2016). Construcción y validación de la Escala de Apreciación de la Calidad del Programa de Asignatura. Propuesta para el estudio de la calidad de la docencia. Formación Universitaria, 9(1), 77-90. http://dx.doi.org/10.4067/S0718-50062016000100009

Juliá, M. T. (2013). Competencias del psicólogo en Chile, propuestas desde las universidades estatales. La Serena, Chile: Editorial Universidad de La Serena. 
Martínez-Molina, A. \& Nistor, A. T. (2016). Evaluación de la calidad docente en la educación superior: un estudio en Chile. En O. M. Arellano (Ed.), Reflexión, formación e investigación en psicología de la educación (pp. 37-47). Santiago, Chile: RIL Editores.

Olate, J. \& Castillo, S. (2016). Desarrollo de procesos reflexivos desde la percepción de estudiantes de enfermería. Revista de Psicología, 25(2), 1-18. http://dx.doi.org/10.5354/0719-0581.2016.44775

Rivera Heredia, M. E., Martínez Fuentes, M., González Betanzos, F., \& Salazar García, M. A. (2016). Autoeficacia, participación social y percepción de los servicios universitarios según sexo. Revista de Psicología, 25(2), 1-16.

http://dx.doi.org/10.5354/0719-0581.2017.44842

Sánchez-Rosas, J. \& Esquivel, S. (2016). Instructional Teaching Quality, Task Value, SelfEfficacy, and Boredom: A Model of Attention in Class. Revista de Psicología, 25(2), 1-20. http://dx.doi.org/10.5354/10.5354/0719-0581.2017.44843

SCImago. (2007). SJR - SCImago Journal \& Country Rank. Consultado el 24 de diciembre de 2016 en

http://www.scimagojr.com

Urzúa, A., Vera-Villarroel, P., Zúñiga, C., \& Salas, G. (2015). Psicología en Chile: análisis de su historia, presente y futuro. Universitas Psychologica, 14(3), 1125-1142. http://dx.doi.org/10.11144/Javeriana.upsy14-3.pcah 